\title{
Technology development of the soft X-ray tomography system in Wendelstein 7-X stellarator
}

\author{
M. Schülke*, A. Cardella, D. Hathiramani, S. Mettchen, H. Thomsen, S. Weißflog, D. Zacharias
}

Max Planck Institute for Plasma Physics, EURATOM Association,

Wendelsteinstr. 1, 17491 Greifswald, Germany

\begin{abstract}
The engineering and design of the soft X-ray Multi Camera Tomography System (XMCTS) in Wendelstein 7-X stellarator (W7-X) must fulfill several additional requirements compared to short pulse machines. The XMCTS has to withstand irradiation and electron cyclotron microwave loads in addition to being ultra high vacuum compatible, having low magnetic permeability and using low neutron activation materials (e.g. Co $\leq 2000 \mathrm{ppm}$ ). A further difficulty is the limited space inside the plasma vessel, which requires special engineering solutions.
\end{abstract}

After detailed design development, supported by finite element analyses, prototypes have been manufactured and tested. At the end all test results have successfully proven that the components fulfill the requirements and that reliable and stable measurements will be possible with the XMCTS diagnostics during W7-X operation.

The paper describes the design and the technological development, in particular on the electric multipin feedthrough (UHV barrier between in vessel detectors and the preamplifiers), the active cooling of the electronic components (reducing dark current/ noise increase), the pneumatic shutter (protection of the detectors from sputtering and during baking) and the fiber optics illumination system (calibration of the detectors).

\section{Introduction}

The new generation fusion devices presently under construction like the Wendelstein 7-X stellarator (W7-X) in Greifswald/ Germany will operate with high power and long discharges of up to 30 minutes. The key goal for $\mathrm{W} 7-\mathrm{X}$ is to show the feasibility of the advanced stellarator concept in a steady-state operation $[1,2]$.

The soft X-ray Multi Camera Tomography System (XMCTS) $[3,4]$ measures the X-ray intensity of the plasma along 380 sightlines utilizing 20 compact pinhole cameras. The data will be used to reconstruct the plasma shape and location by means of tomographic inversion.

\section{Requirements}

The development and integration of diagnostic components for the fusion plasma experiment Wendelstein 7-X stellarator is demanding in terms of design and engineering due to the steady state operational requirements. In the following, some of the challenges and solutions are shown for the XMCTS. The requirements result from the operational scenarios of $\mathrm{W} 7-\mathrm{X}$ as well as the diagnostics functions.

The general requirements for components inside magnetic confinement fusion devices are:

- Heat resistance (plasma convection and radiation, conditioning discharges, microwave)

- Radiation hardness (neutrons)

- Ultra high vacuum (UHV) compatibility $\left(<10^{-8} \mathrm{mbar}\right)$

*Corresponding author

Email address: mathias.schuelke@ipp.mpg.de (Mathias Schülke)
- Low magnetic permeability $\left(\mu_{\mathrm{r}} \leq 1.01\right)$

- Resilience against plasma sputtering

- Long life time of components (> 15 years)

- Small installation space

Due to the long pulse operation, the radiation hardness and steady state heat loads are of special concern for the design of in-vessel components in the W7-X stellarator. Additionally, special requirements are given by the two main functions of the cameras (Fig.1), the spatially resolved measurement of soft-X rays and the calibration of the detectors. For the former function, a good signal quality (low noise, high bandwidth) is important, whereas a spectral resolution is not required. The soft- $X$ ray radiation is measured by a silicium diode detector, which converts the radiation into small electrical signals $(\mathrm{nA}-\mu \mathrm{A})$. Since this type of detector is also sensitive to visible light, a $10 \mu \mathrm{m}$ thick beryllium foil is used as a filter. In order to achieve a low noise, high bandwidth signal, a preamplifier close to the detector is mandatory, which drives the lines to the data acquisition system outside the plasma vessel (distance $\sim 15 \mathrm{~m}$ ). The detector and the preamplifier electronics are temperature sensitive, temperatures above $T_{\mathrm{c} 1}=40{ }^{\circ} \mathrm{C}$ should be avoided, since the dark current level increases exponentially with temperature [3]. A hard temperature limit for the preamplifier circuit is $T_{\mathrm{c} 2}=100{ }^{\circ} \mathrm{C}$, above which some electronics components (capacitors, op-amps) will fail. The detector can be operated in vacuum, but the preamplifier components are not UHV compatible. Hence, the preamplifier must be separated from the vacuum in the W7-X plasma vessel. 
This is realized by a vacuum tight stainless steel box (electronic box) including the preamplifier. The electric contacts for the signal transport from the detector to the amplifiers is realized by a custom made vacuum feedthrough with plug connectors on both sides.

The spatial resolution of one of the soft-X ray cameras is achieved by separated sensitive areas in one direction of the detector in conjunction with a pinhole. Each detector element collects soft-X ray radiation along a different sight cone from the plasma through the pinhole and the filter. No light from other directions (i.e. not entering the pinhole) should be able to reach the detector elements. This leads to the competing requirements of a light-tight (and ECRH stray radiation tight) volume shielding the detector from stray light and a vacuum compatible design, i.e. the volume must be pumpable. The beryllium foil and the filter layer protect the detector from the Electron Cyclotron Resonant Heating microwave $(\mathrm{ECRH})$ stray radiation.
The second main function of the XMCTS is the calibration of the detector elements by means of a light source. The detector elements are illuminated by a light source of known frequency and intensity. The light source is located outside the plasma vessel and the light is guided by a fibre optics cable to the detector (bypassing the filter). Another calibration option is foreseen during long pulse discharges. The X-ray radiation through the pinhole should be blocked in order to calibrate the zero offset of the detector and the preamplifier during operation with possibly increased temperatures. This is realized by a pneumatic shutter in front of the pinhole. Additionally, this shutter can protect the filter from sputtering during cleaning discharges and baking of the W7-X plasma vessel. This is expected to increase the life time as well as the measurement signal quality.

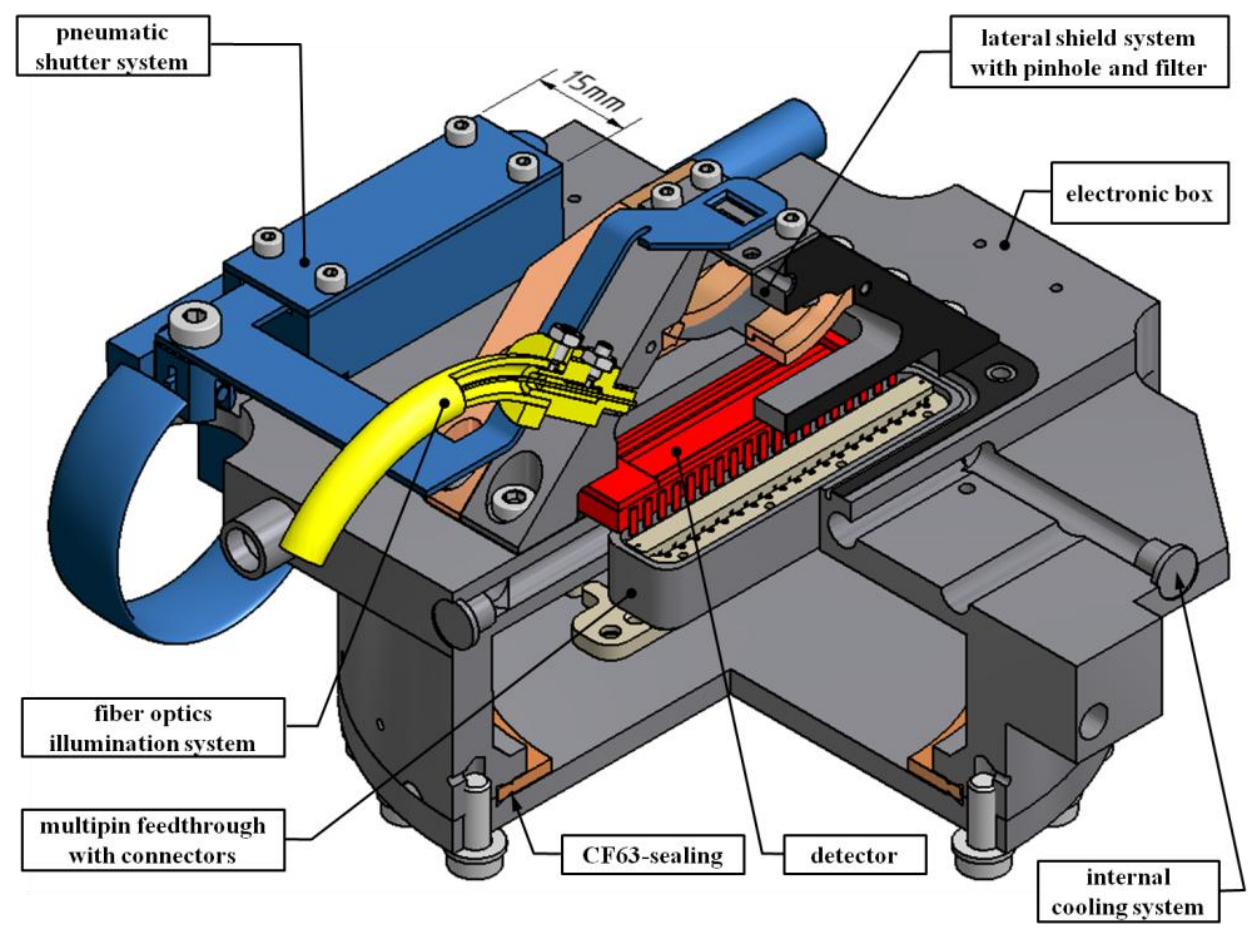

Fig. 1 The sectional exposition of the XMCTS camera with an overview of the main components. (One cooling side plate is removed and several parts are cut away to expose the details, e.g. the water cooling circuit.)

\section{Solutions}

In the following, we present engineering solutions achieving the set of complex requirements for exemplary challenges.

\subsection{Internal cooling system}

One major design challenge is the compliance with the temperature limit of detector and preamplifier.

During long plasma discharges (up to $30 \mathrm{~min}$ ), this requirement can only be met by active cooling. The engineering options are limited, in particular due to the severely restricted space behind the plasma facing components. These components may sustain heat loads up to $500 \mathrm{~kW} / \mathrm{m}^{2}$ transiently and $250 \mathrm{~kW} / \mathrm{m}^{2}$ in steady state, and may reach temperatures up to $450{ }^{\circ} \mathrm{C}$.

Finite element method (FEM) calculations were used to optimize the layout of the water cooling of the electronic box. The sufficient cooling of the detector could mainly be achieved by cooling channels drilled into the head of the box with one channel located directly underneath the detector (Fig.2). The boreholes are sealed at the ends by welded plugs and form a meandering path for the cooling water flow. In contrast to a solution with external cooling pipes, the heat transmission between different materials / components is improved and an evenly good contact along the windings is ensured. These optimizations made it possible to use stainless steel instead of higher conductivity materials as 
$\mathrm{CuCrZr}$ which would have complicated the engineering. This is very important for the vacuum-tight welding of the feedthroughs into the electronic box, because of the complexity in welding technology needed to connect two different materials with different physical properties (heat conductivity, thermal expansion, local restraint) such as copper and stainless steel. The cooling side plates are made from $\mathrm{CuCrZr}$ in order to achieve heat conduction as good as possible. The thermal contact between the cooling side plates, the camera body and the electronic box is improved by an interlayer of $0.15 \mathrm{~mm}$ graphite foil, ensuring an evenly distributed heat contact [6].

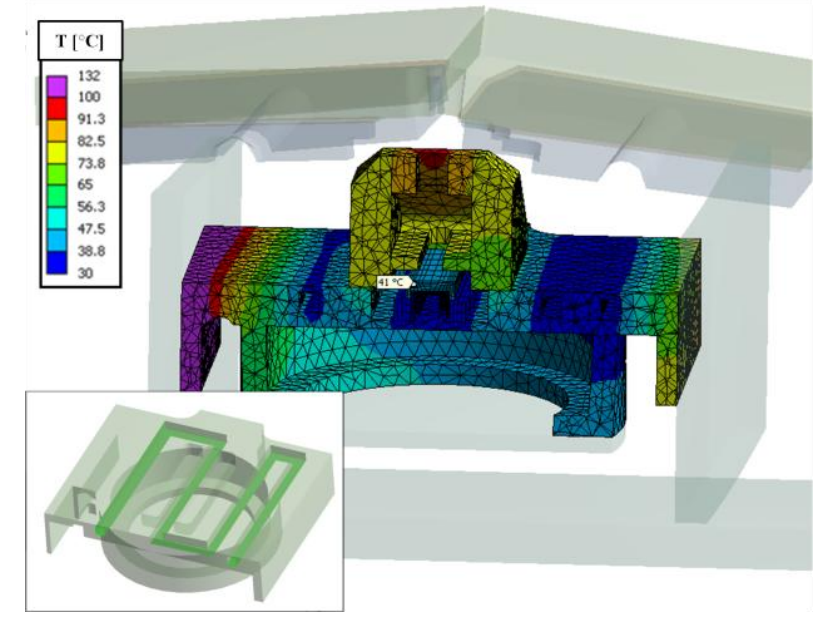

Fig.2 FEM simulation of the temperature distribution of the XMCTS camera during operation of the W7-X. The optimized routing of cooling water is shown in the bottom left inset. For simulation of the routing rectangular pipes are shaped and in reality the rectangular pipes are replaced by $5 \mathrm{~mm}$ boreholes.

\subsection{Mulipin feedthrough with connectors}

The electrical signals from the detector are sent to the preamplifier inside the electronic box via a custom-made multipin feedthrough, which ensures that the volume inside the box is UHV separated from the vacuum inside the plasma vessel. One feedthrough consists of a stainless steel base body with 19 drilled holes. Inconel pins are glazed into each of these holes. This feedthrough is welded into a notch inside the electronic box (Fig.3). During the welding process, the temperature of the glass around the pins should not exceed $250^{\circ} \mathrm{C}$, otherwise there is a risk of leakages.

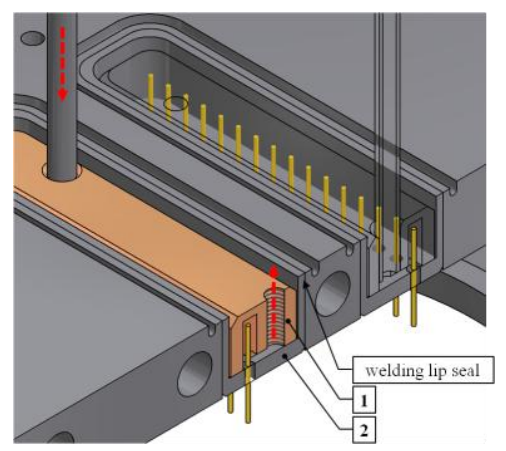

Fig.3 Detailed view of the multipin feedthrough (2) with the temporary inserted copper protection cap (1). The dashed line arrow shows the forming gas flow. (The right copper protection cap is illustrated semi-transparent)
This requirement was assured by a number of design and technological measures: (1) the material combination is stainless steel with stainless steel in the electronic box. (2) The U-shape form of the base body increases the distance between welding zone and the delicate glazing and minimizes the cross-section for heat conduction. (3) The welding lip seal was used as weld seam geometry due to the comparatively low heat input. (4) The welding of one multipin feedthrough is interrupted (6 times) to avoid overheating. (5) A forming gas was cooling the feedthrough under a copper protection cap, which protects the pin against heat radiation.

For the electrical contact between the detector and the preamplifier, two additional connectors, one outside (primary connector) and one inside (secondary connector) the electronic box are necessary. It is not possible to use standard plugs due to the small available space in vertical direction. That's why horizontal connection adaptors had to be developed (Socket Factory GmbH, Greding/Germany). The primary connector (Fig.4) must fulfill the vacuum requirements. It consists of a PEEK ${ }^{\circledR}$ housing with two parallel rows of drilled holes. The first row of holes holds the connectors of the detector, the second row the pins of the feedthrough. Each pair of holes is electrically connected via a gold coated contact spring in $\mathrm{W}$-shape, which is manufactured by an etching process (Fig.4, top left insert). The ends of the spring establish the contact. A special unlocker inserted into the primary connector presses the springs together and interrupts the connections during installation.

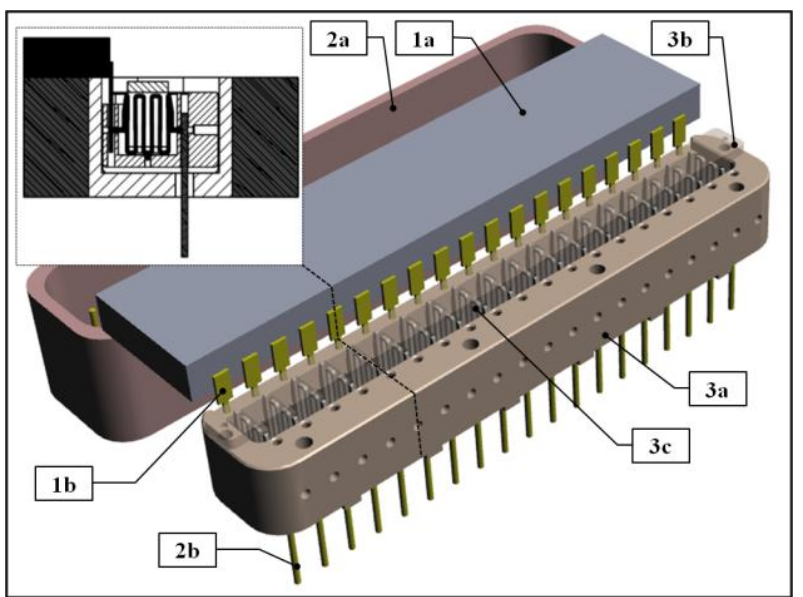

Fig.4 A detailed view of the multipin feedthrough $(2 a, b)$ in connection with the primary connector $(3 a, b, c)$ and the detector (1a). The primary connector is totally inserted into the base body (2a) of the feedthrough and connects the detector pins (1b) and the pins of the multipin feedthrough (2b). Each detector needs two multipin feedthroughs, one for each pin row of the detector. The sectional view (top left) shows the special form of the contact spring (3c), which is fixed by a PEEK housing (3a) and a bar (3b, semi-transparent).

\subsection{Lateral shield system}

The sketch in Fig. 5 illustrates the pinhole arrangement of the XMCTS camera body and the detector. The bent beryllium foil within the filter layer keeps out the visible light and shields the detector from 
ECRH stray radiation. The volume between detector and filter (Fig.5, Volume 2) needs to be pumped in order to be compatible with the vacuum requirements. This requirement is solved by two rows of drilled holes inside the cooling side plates. The additional requirement to avoid ECRH stray radiation at the detector leads to a maximum acceptable hole diameter of only $0.7 \mathrm{~mm} \mathrm{[5].}$ The detector should not receive any direct light, expected from the pinhole / filter combination. This is achieved by placing one row of holes well below the active detector elements. The second row of holes (Fig.5, broken circle) located above the detector is connected to the outside vacuum with a larger borehole perpendicular to the multiple small holes. Thus, the pumping of the volume is achieved, but any light from an arbitrary direction outside the volume has no straight line into the detector volume. Light can reach the detector elements only with multiple reflections, which will reduce its intensity and the measurement perturbation to a negligible level. Gaps at the junctions between two metal components (e.g. between the cooling side plates and the camera body) all have an interlayer of graphite foil, which effectively shields incoming light and ECRH stray radiation as well as improving the thermal contact. Moreover, the tolerances of some mechanical parts are very small. For example the maximal gap between the camera body and the filter layer, which is pushed into the camera body (Fig.6 top right), is 0 to $0.04 \mathrm{~mm}$.

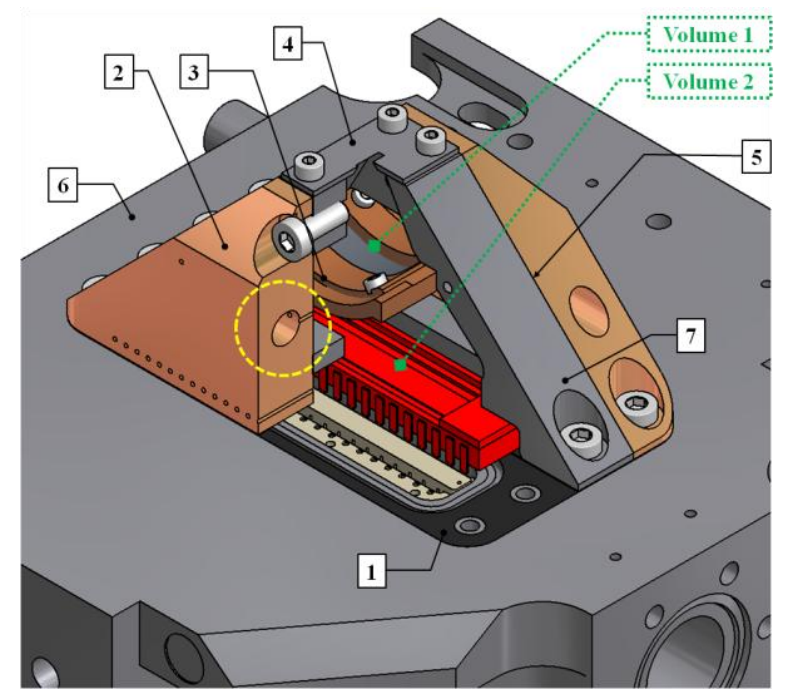

Fig.5 A sectional view into the lateral shield system with pinhole (4) and camera body (7), which is bolted on the electronic box (6). For a better thermal transport and a good microwave and light tightness a graphite foil $(1,5)$ is placed under and between the parts of the lateral shield. The cooling side plates (2) are made of $\mathrm{CuCrZr}$ and the camera body of stainless steel. The beryllium filter layer (3) separates two volumes, which have to be pumpable.

\section{Tests}

Every developed solution presented in section 3 was successfully checked for its functional and operational serviceability. For example the developed welding technology applied to the cooling system and the feedthroughs was qualified by helium leak tests, macroexamination specimens and visual inspections starting with some simple mockups and ending with a qualified series specimen. The pneumatic shutter underwent tests in vacuum over more than $10^{5}$ cycles to prove the long term stability and to determine, how the different parts react after so many working cycles. An increase of friction or fatigue was not observed.

A special microwave experimental facility MISTRAL to test the ECRH stray radiation compatibility [5] was utilized for checking the camera closure. A complete camera prototype including electronics box, pinhole, filter and cooling side plates was tested (Fig.6). The detector was simulated by a PEEK block of similar size, which was instrumented with a thermocouple to measure the temperature increase (Fig.6, top right insert). The electronic box and one cooling side plate were additionally instrumented each with one thermocouple.

The test showed that the applied solution is compatible with the expected level of ECRH stray radiation. The temperature increase in the PEEK block was similar to the temperature increase of the measuring point of the electronic box and well below the critical temperature.

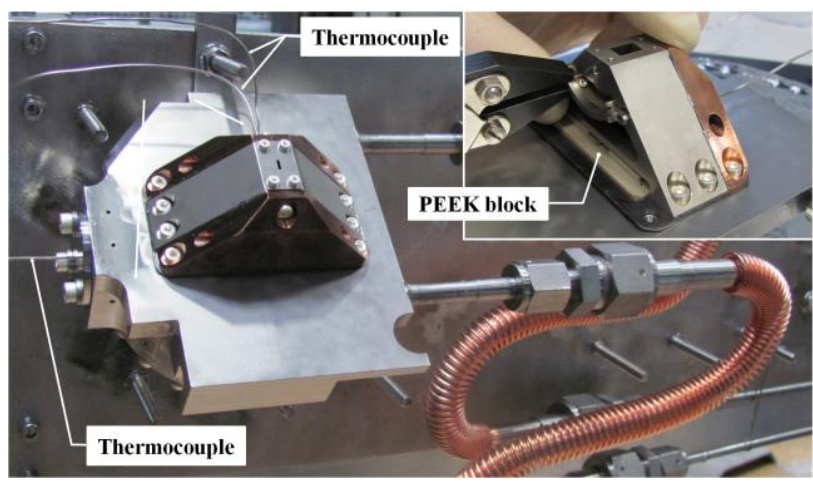

Fig.6 XMCTS camera mockup for a long-term thermal load test in MISTRAL.

\section{Conclusion}

The development of the XMCTS illustrates the complex requirements the $\mathrm{W} 7-\mathrm{X}$ project poses to invessel diagnostics and how they can be successfully fulfilled. Especially the camera of the XMCTS demonstrated in many tests, that stable and reliable measurements will be possible in the future operation of the Wendelstein 7-X stellerator.

\section{References}

[1] R. König et al., Review of scientific instruments 83, 10D730 (2012).

[2] R. Burhenn et al., Contrib. Plasma Phys. 51, SI271 (2011).

[3] A. Weller et al., Review of scientific instruments 75, 3962 (2004).

[4] H. Thomsen et al., AIP conference proceedings 993, 163 (2008).

[5] D. Hathiramani et al., submitted for publication to Fus. Eng. Des. (2012), Contribution to the $27^{\text {th }}$ Symposium on Fusion Technology (SOFT)

[6] H. Greuner et al., Fus. Eng. Des. 66 - 68 (2003) 447 - 452 